\title{
Introducción al concepto de hábito de Charles Peirce para el comportamiento del consumidor
}

Fecha de recepción: 11 de marzo de 2011

Fecha de aprobación: 13 de mayo de 2011

\begin{abstract}
Alejandro Castaño Ramírez
acastano@poligran.edu.co

Politécnico Grancolombiano

Antropólogo de la Universidad de los Andes, coordinador de investigaciones de mercadeo en Whitney International University System.
\end{abstract}

\section{Resumen}

La disciplina del conocimiento del consumidor ha hecho uso de un concepto de hábito que parte, en gran medida, del sentido común. Sin embargo, hay diversas aproximaciones al hábito que pueden dar nuevos caminos para la adecuada comprensión del comportamiento del consumidor. El concepto de hábito de Charles S. Peirce formula ideas aprovechables para el mercadeo y permite analizar el hábito desde una perspectiva informada y enriquecida.

\section{Palabras clave}

Hábito, Charles Peirce, comportamiento del consumidor.

\begin{abstract}
The discipline of consumer knowledge has used a habit concept that comes, mostly, from common sense. However, there are several approximations to the habit that could guide us in order to get a better understanding of the consumer behavior. The habit concept elaborated by Charles S. Peirce, establishes a concept useful for marketing and allows analyzing the habit from an informed and enriched approach.
\end{abstract}

\section{Keywords}

Habit, Charles Peirce, Consumer Behavior. 
Entre los conceptos más fértiles en el conocimiento del consumidor y el mercadeo está el de hábito. Este concepto ha permitido la comprensión de las rutinas de consumo y el panorama de productos que giran a su alrededor; ha permitido establecer argumentos alrededor del ciclo de vida del producto, de innovaciones y de cambios culturales para anticipar tendencias.

Sin embargo, su aproximación se hace, en la mayoría de los casos, desde el significado que le da el sentido común, lo que puede deberse al amplio uso cotidiano del término. La teoría de los signos de Charles Peirce aborda el concepto de hábito de diversas formas, tales como la de interpretación y acción. Además, entre otros postulados, plantea un dinamismo en los hábitos en la medida en que estos son adquiridos, crecen, se perfeccionan y llegan a ser abandonados.

En el presente ensayo analizaremos dos de dichos postulados: primero, la conceptualización del hábito en crecimiento; segundo, una perspectiva no consciente pero racional que le permitirá a la disciplina del conocimiento del consumidor pensar el hábito de una manera diferente.

\section{El hábito en crecimiento}

En la extensa obra de Charles Peirce se usa el concepto de hábito en una gran variedad de maneras. Gary Shapiro identifica algunas de ellas: "creencias, principios lógicos, disposiciones, instintos y personalidad" (2003, p. 26).

No obstante, de acuerdo con Shapiro, Peirce tiene dos maneras de usar el concepto que se destacan entre las otras por ser más frecuentes. Primero está aquella en la que el hábito es una disposición a actuar de una determinada manera, y esa determinación está impulsada por un propósito. La segunda entiende el concepto por su generalidad, es decir, que se obtendrá una determinada clase de resultado si la ocasión es adecuada, aunque dicha determinación tiene condiciones y no es absoluta (2003).

Enfocándonos en la primera forma frecuente de uso, en la que el hábito en Peirce es entendido como una disposición a actuar de una manera cuya determinación es impulsada por un propósito, obtendremos que uno de los caminos posibles para entender los hábitos, dentro del conocimiento del consumidor, es analizarlos a partir de lo que los motiva y no solamente como un patrón de comportamiento.

Así, el hábito deja de lado el paradigma conceptual cotidiano que opera como aquella fuerza, natural o social, que ciñe o rige el comportamiento, volviéndose un factor que condiciona la misma acción que lo incita. A esto se suma que todo comportamiento es significativo debido a que busca un propósito (Shapiro, 2003). Lo que implica que, metodológicamente, la investigación del consumidor debe dar cuenta de los detalles del comportamiento, haciendo necesarias las herramientas que permitan conceptualizar lo que pocas veces expresamos.

Esto que hemos señalado hasta el momento sobre el hábito como acción no corresponde en Peirce solamente a la acción física, es decir, al hábito que implica una acción del cuerpo, sino que también corresponde al hábito del pensamiento, que surge del ejercicio de la imaginación.

Estos tipos de hábitos de interpretación son conceptos que aparecen como interpretantes finales, como referencias 
que -sin tener que entrar a definir todos los elementos que conforman una cadena de interpretantes- permiten el flujo de interpretación de la realidad.

Las inferencias y la inducción serían otro tipo de hábito de interpretación, en la medida en que implica un hábito en la forma de pensar, un procedimiento (Shapiro, 2003; Niño, 2008). Este procedimiento es establecido y, a su vez, establece reglas.

La permanencia y el cambio de estas reglas afectan directamente una ley de Peirce que es resaltada por Vincent Colapietro (2009): "la ley de crecimiento" (ep 2, 445). De acuerdo con ella, los hábitos crecen en el comportamiento, así como también decrecen y cambian. El hábito, por lo tanto, es móvil, no se adquiere de una vez para toda la vida, sino que está en constante actualización. Incluso podemos afirmar, como lo hace Vincent Colapietro (2009), que los hábitos promueven cambios y son grandes fuerzas de influencia. Esto es absolutamente aprovechable desde la perspectiva del mercadeo y la persuasión publicitaria en la medida en que el mercado se desarrolla y se instauran nuevas necesidades.

La dinámica social de los hábitos se puede asociar en Peirce al segundo de los usos más frecuentes del concepto hábito que nos indica Shapiro, (2003) y que es resaltado también por Colapietro (2009): la característica del hábito de ser general, o sea, que se puede decir lo mismo de él en diferentes contextos (Colapietro, 2009). Se considera que este concepto está directamente relacionado con la dinámica social de los hábitos porque establece la coherencia y la estabilidad, las cuales permiten espacios de comunicación social. La riqueza del hábito nos habla, entonces, de una historia de éstos, la cual nos puede explicar las maneras en que el ser humano se adapta a su medio ambiente natural y genera una cultura.

La identificación de procedimientos mentales de abstracción y abducción, y la rigidez de la designación (Short, 2007), aplicadas a la interpretación de la explicación de las formas de comportamiento de las personas, generalmente obtenidas en discursos cualitativos, nos pueden develar la manera como se asocian los signos y los elementos que los componen. La abstracción y la abducción nos explicarían la manera en que se introducen nuevas asociaciones de signos a un discurso, mientras que la rigidez de la designación nos indica aquello que es central a un signo.

\section{Dimensiones del hábito: no consciente pero racional}

La disciplina del conocimiento del consumidor se mueve en un paradigma que indica que quien consume lo hace de manera predominantemente inconsciente. A esto se llegó luego de la revisión del concepto de homus economicus, que indicaba una racionalidad en las formas de consumo en el ser humano. Ese concepto fue reemplazado por otros a través de publicaciones de economistas que consideran que el consumo ocurre $95 \%$ de manera subconsciente y/o inconsciente y un 5\% de manera consciente (Zaltman, 1995); y del trabajo de consultores empresariales que asocian el comportamiento de consumo con la teoría del cerebro triúnico, en la que dichos comportamientos están anclados al cerebro reptil y suceden de manera instintiva (Rapaille, 2007).

De acuerdo con Colapietro (2009), debido a su búsqueda de analizar aquellos 
hábitos que están relacionados con la ciencia, el pensamiento de Charles Peirce sobre el hábito se centra en aquellos que son adquiridos deliberadamente. Pero eso no le cierra la puerta a otros tipos de hábitos que pudo conceptualizar Peirce (Colapietro, 2009). La diferencia entre tipos de hábitos, especialmente con relación a los opuestos dicotómicos deliberados/no conscientes, es solamente una entre las muchas posibles. Se encuentran, además, diferencias entre hábitos de acción e interpretación, lógicos y emocionales, y con alta o baja integración del hábito al comportamiento, entre otras que surgen a partir del modelo triádico del signo y otros conceptos como el grado de claridad, propuestos por Charles Peirce.

Es indispensable apuntar que, para Peirce, el dualismo no tiene un radical en el sentido de oponer polaridades absolutas y tajantes, sino que aquellos opuestos aparentes pueden estar casi encarnados el uno con el otro, en una conexión inseparable (Shapiro, 2003). Esta forma de pensar es denominada "sinequismo", y busca el análisis y comprensión de la realidad a través de continuidades antes que divisiones. Así, se crea conceptualmente un continuo de hábitos que se ubica a lo largo de estas polaridades.

Para el conocimiento del consumidor, el comportamiento inconsciente y/o subconsciente es el que predomina en el consumo. En Peirce podemos ampliar esta conceptualización en la medida en que define aquellos hábitos no deliberados como "unreflectively established habits", los cuales están ampliamente relacionados con un sentido práctico (Colapietro, 2009, p. 552). Éste no debe tomarse únicamente como una respuesta automática de un ser vivo, sino que también obedece a las "dispositionally improvisational exertions" (Colapietro, 2009, p. 552); implica adaptaciones de los organismos a las condiciones del mundo que los rodea, por ejemplo, adaptaciones al frio o al calor.

Así, a través del sentido práctico de pensar el hábito con relación a su propósito, encontramos un camino para dar razón de aquella etapa inconsciente y/o subconsciente en el consumo, considerando los grados de claridad con una herramienta útil para obtener este objetivo.

Dentro de las dimensiones del hábito hay un escenario que puede llegar a ser fructífero para el conocimiento del consumidor. Y es que, para Peirce, el concepto de hábito tiene un tipo que es simbólico (Short, 2007). Por su parte, Winfried Nöth, quien escribe sobre el símbolo y el hábito, dice que el tipo de signo que son los símbolos se crea a partir de su interpretación, independientemente de una similitud o conexión real entre el "representamen" y el "objeto dinámico". Por lo tanto, son hábitos, e incluso reglas, de interpretación (Nöth, 2010; Short, 2007).

Un símbolo, entonces, al determinar su interpretante por una regla y su objeto dinámico por un interpretante, es convencional y puede generar nuevos objetos dinámicos e interpretantes en un ciclo de vida (Nöth, 2010; Short, 2007). Esto abre una discusión alrededor del tema de la posible reproducción de los símbolos que son creados por sus usuarios, pero que también crean en el usuario una realidad. En este sentido, la interacción que existe entre los seres vivos y los símbolos que son usados por ellos tiene también la forma de una simbiosis, en la que existe un sistema de interacción.

Estos sistemas pueden asociarse a dos tipos de círculos en los que giran los símbolos con quienes los usan, identificados 
por Winfried Nöth como el vicioso y el virtuoso, diferentes por el crecimiento semiótico mayor del segundo con respecto al primero (2010).

\section{Aplicaciones al conocimiento del consumidor}

De los conceptos asociados al hábito en Peirce que se han presentado, señalaremos, a continuación, algunas aplicaciones al conocimiento del consumidor, que se suman a las ya mencionadas.

La naturaleza cambiante de los hábitos y su crecimiento a partir de una cadena de interpretantes apuntan hacia el estudio de los hábitos desde una perspectiva dinámica, en la que la rigidez tiene validez como frecuencia pero no mecanismo absoluto de dirección de la acción. Así, se puede establecer una tabla de clasificación de crecimiento de un hábito y entender las razones y significados de los ciclos de vida de un producto o una tendencia y la forma de integrar y motivar comportamientos en la sociedad.

El concepto de hábito como propósito también es aplicable al conocimiento del consumidor en tanto que nos ayuda a establecer las razones que motivan aquellos hábitos que son inconscientes y/o subconscientes. Los grados de claridad son una herramienta para lograr determinar la naturaleza de este tipo de hábitos, en la medida en que supone un proceso de reconocimiento casi que auto-reflexivo.

En la clarificación, al definir un concepto, debo traducirlo en otros, moviéndome a lo largo de una cadena de interpretantes. Por lo tanto, identificar los signos a través de esta cadena de interpretantes nos puede dar nociones claras de los propósitos que condicionan el consumo de una persona o una comunidad.

Conceptos como la abstracción y la abducción nos pueden mostrar la manera como en un discurso un agente construye un significado alrededor del consumo de un producto o servicio. El concepto de rigidez de la determinación ayudará a caracterizar las rutas de significado y los elementos centrales de éstas.

De los postulados sobre el hábito surge una hipótesis a verificar que también es útil al conocimiento del consumidor y el mercadeo, a saber: en la medida en que el hábito sea más deliberado, éste puede ser más modificable, puesto que tiene un mayor grado de claridad; de manera opuesta, aquellos hábitos menos clarificados pueden ser los menos modificables y los que necesiten un mayor trabajo por parte del mercadeo y la publicidad para su modificación.

En una línea diferente, la perspectiva del símbolo como un hábito de interpretación se puede asociar con la marca de los productos y servicios como espacio de aplicación del conocimiento del consumidor. En este contexto, la marca sería un símbolo que funciona como objeto dinámico y establece sus propios interpretantes. La clasificación de Winfrid Nöth de los dos tipos de círculos genera un modelo para la medición de la salud de marca y establecer el brand equity -0 valor de la marca-.

Al postular una transición gradual, una continuidad, entre opuestos (naturaleza y cultura, materia y vida), la doctrina del sinequismo de Peirce está relacionada con estos tipos de círculos. En esa medida, el símbolo, y en este caso específico la marca-símbolo, le enseña al 
hombre y éste comprende el mundo a través de ella.

Cada símbolo crece dentro de un campo simbólico, en el que se adapta a un espacio (mental-cultural) y puede crecer si está en un círculo virtuoso. ¿Cuáles son estos universos simbólicos en los que se insertan las marcas?, es una pregunta válida para el mercadeo.

Otra aplicación del concepto de hábito, como es propuesto por Peirce, dentro de un escenario de interpretación, le será útil al conocimiento del consumidor. $Y$ es la identificación de hábitos en las formas de pensar, en la medida en que le permitirán a éste caracterizar funciones como la seducción o la persuasión.

El presente ensayo es una introducción al concepto de hábito en Charles Peirce, central a su forma de pensamiento sobre los signos y la comunicación. Profundizar en este concepto puede abrir nuevas oportunidades para el desarrollo del conocimiento del consumidor y de aplicaciones al mercadeo y la publicidad.

\section{Referencias}

I. Colapietro, V. (2009). Habit, Competence and Purpose: How to Make the Grades of Clarity Clearer. Transactions of the Charles S. Peirce Society, 43 (3), 348-377.

2. Niño, D. (2008). El signo peirceano y su impacto en la semiótica contemporánea. En Ensayos semióticos (pp. 15-100). Bogotá: Universidad Jorge Tadeo Lozano.

3. Nöth, W. (2010). The Criterion of Habit in Peirce's Definition of the Symbol. Transactions of the Charles S. Peirce Society, 46 (1), 82-93.

4. Rapaille, R. (2007). El código cultural. Bogotá: Editorial Norma.

5. Short, T. (2007). Peirce's Theory of Signs. Cambridge: Cambridge University Press.

6. Shapiro, G. (1973). Habit and Meaning in Peirce's Pragmatism. Transactions of the Charles S. Peirce Society, (9) 1, 24-40.

7. Zaltman, G. (2003). How Customers Think. Boston: Harvard Business School Press. 